\title{
Survival Probability of a Local Excitation in a Non-Markovian Environment: Survival Collapse, Zeno and Anti-Zeno effects.
}

\author{
E. Rufeil Fiori ${ }^{1, *}$ and H. M. Pastawski ${ }^{1, \text {, }}$ \\ ${ }^{1}$ Facultad de Matemática, Astronomía y Física, and Instituto de Física (CONICET), \\ Universidad Nacional de Córdoba, Ciudad Universitaria, 5000, Córdoba, Argentina.
}

\begin{abstract}
The decay dynamics of a local excitation interacting with a non-Markovian environment, modeled by a semi-infinite tight-binding chain, is exactly evaluated. We identify distinctive regimes for the dynamics. Sequentially: (i) early quadratic decay of the initial-state survival probability, up to a spreading time $t_{S}$, (ii) exponential decay described by a self-consistent Fermi Golden Rule, and (iii) asymptotic behavior governed by quantum diffusion through the return processes and leading to an inverse power law decay. At this last cross-over time $t_{R}$ a survival collapse becomes possible. This could reduce the survival probability by several orders of magnitude. The cross-overs times $t_{S}$ and $t_{R}$ allow to assess the range of applicability of theFermi Golden Rule and give the conditions for the observation of the Zeno and Anti-Zeno effect.
\end{abstract}

PACS numbers:

\section{INTRODUCTION}

The decay of an unstable local state is usually described, within a Markovian approximation, by an exponential decay with a rate given by the Fermi Golden Rule (FGR). However, this description contains approximations that leave aside some intrinsically quantum behaviors [1]. Indeed, works on models for nuclei, composite particles [2] and excited atoms [3], predict that the exponential decay does not hold for very short and very long times, and this exponential decay may shows superimposed beats.

In Ref. [4] we presented an exactly-solvable model describing the evolution of a surface excitation in a semiinfinite chain. Physical realizations of one-dimensional systems are provided by electron transport in superlattices [5], discrete diffraction in photonic crystals [6], and spin excitations in a chain of nuclear spins under an XY interaction [7] or under double quantum interaction [8]. Here, we quantify and interpret the short and long time limits, $t_{S}$ and $t_{R}$, of the FGR. We identify three time regimes for the survival probability $P_{00}(t)$. Initially the decay is quadratic and it holds up to a time $t_{S}$. From $t_{S}$ to $t_{R}$ it is exponential, and finally, for long times, it follows a power law. The time $t_{S}$ gives an upper bound to the time interval at which repetitive projection measurements could lead to a Quantum Zeno Effect ([9], [10]). On the other hand, at $t_{R}$, a dip in $P_{00}(t)$ of several orders of magnitude may occur. This survival collapse is identified with a destructive interference between the pure survival amplitude, i.e., an exponential decay amplitude, and a return amplitude, which is usually neglected because it arises from memory effects in the environment. This destructive interference can be used to obtain an anti-Zeno effect [11], where the decay rate is strongly enhanced by

\footnotetext{
*Electronic address: rufeil@famaf.unc.edu.ar
}

${ }^{\dagger}$ Electronic address: horacio@famaf.unc.edu.ar repeated projective measurements with period $t_{R}$.

\section{SURVIVAL PROBABILITY}

The evolution of a state $|0\rangle$ weakly coupled to a set of states which defines the "environment", is described by the survival probability

$$
\begin{aligned}
P_{00}(t) & =|\langle 0|\exp [-\mathrm{i} \mathcal{H} t / \hbar]| 0\rangle \theta(t)|^{2} \\
& \equiv \hbar^{2}\left|G_{00}^{R}(t)\right|^{2}, \\
& =\hbar^{2}\left|\int \frac{\mathrm{d} \varepsilon}{2 \pi \hbar} G_{00}^{R}(\varepsilon) \exp [-\mathrm{i} \varepsilon t / \hbar]\right|^{2}, \\
& =\left|\theta(t) \int_{-\infty}^{\infty} \mathrm{d} \varepsilon N_{0}(\varepsilon) \exp [-\mathrm{i} \varepsilon t / \hbar]\right|^{2},
\end{aligned}
$$

where $G_{00}^{R}(t)$ is the retarded single particle Green's function and $N_{0}(\varepsilon)$ is the Local Density of States (LDoS). This is evaluated expanding the initial condition in the eigenstates $|k\rangle$ of the Hamiltonian $\mathcal{H}$, or by using the energy representation of the Green's function $G_{00}^{R}(\varepsilon)$,

$$
\begin{aligned}
N_{0}(\varepsilon) & \equiv \sum_{k}|\langle 0 \mid k\rangle|^{2} \delta\left(\varepsilon-\varepsilon_{k}\right), \\
& =-1 / \pi \operatorname{Im} G_{00}^{R}(\varepsilon) .
\end{aligned}
$$

If the spectrum is bounded, Eq. (4) can be calculated using the residue theorem with the path shown in Fig. 1)

Resonances appear as poles of the analytical continuation $N_{0}(z) \equiv N_{0}\left(\varepsilon+\mathrm{i} \varepsilon^{\prime}\right)$ in the lower complex semi-plane. A well defined resonance appears when an initially unperturbed state of energy $\varepsilon_{0}=\langle 0|\mathcal{H}| 0\rangle$, far enough from the band-edge, is weakly coupled to a continuum, i.e., the expansion of $|0\rangle$ in terms of the eigenstates has a small breath $\Gamma_{0}$ around an energy $\varepsilon_{r}=\varepsilon_{0}+\Delta_{0}$, where $\Delta_{0}=\Delta\left(\varepsilon=\varepsilon_{r}\right)$ is a small shift due to the interaction. This condition excludes out-of-band resonances, virtual 


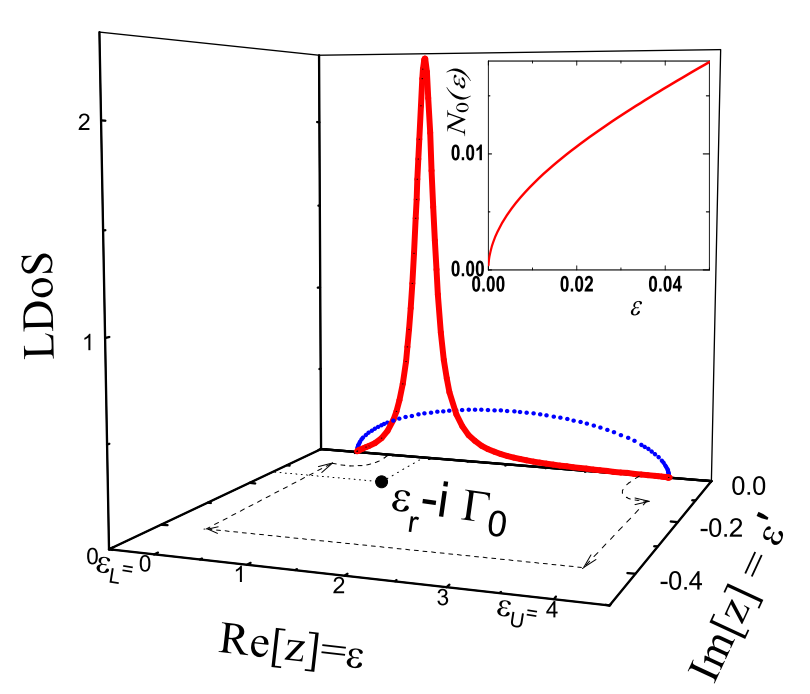

FIG. 1: (color online) Local Densities of States (LDoS) in the complex plane $z=\varepsilon+\mathrm{i} \varepsilon^{\prime} . \quad \varepsilon_{L}$ and $\varepsilon_{U}$ are the lower and upper band-edges, respectively. The solid line is $N_{0}(\varepsilon)$ for a semi-infinite chain with a surface impurity. The dotted line is $N_{1}^{(0)}(\varepsilon)$ for a semi-infinite homogeneous chain, and in the inset is shown the lower band-edge of $N_{0}(\varepsilon)$. The pole appears in $\varepsilon_{r}-\mathrm{i} \Gamma_{0}$. The integration path is shown with dashed lines; it consist of four straight lines and two arcs (which avoid the band-edges singularities).

states and localized eigenstates [15]. Then,

$$
\begin{aligned}
P_{00}(t) & =\mid \underbrace{a \mathrm{e}^{-\left(\Gamma_{0}+\mathrm{i} \varepsilon_{r}\right) t / \hbar}}_{\mathrm{SC}-\mathrm{FGR}} \\
& +\int_{0}^{\infty} \mathrm{e}^{-\varepsilon^{\prime} t / \hbar}\left[\mathrm{e}^{-\mathrm{i} \varepsilon_{L} t / \hbar} N_{0}\left(\varepsilon_{L}-\mathrm{i} \varepsilon^{\prime}\right)\right. \\
& \left.\underbrace{\left.-\mathrm{e}^{-\mathrm{i} \varepsilon_{U} t / \hbar} N_{0}\left(\varepsilon_{U}-\mathrm{i} \varepsilon^{\prime}\right)\right] \mathrm{d} \varepsilon^{\prime}}_{\text {return correction from quantum diffusion }}\right|^{2},
\end{aligned}
$$

where $a=2 \pi \mathrm{i} \lim _{z \rightarrow \varepsilon_{r}-\mathrm{i} \Gamma_{0}}\left[\left(z-\varepsilon_{r}+\mathrm{i} \Gamma_{0}\right) N_{0}(z)\right]$ is the pole residue. $P_{00}$ presents two separate contributions for the decay. The first term (the pole contribution) of Eq.(7) supersedes the usual FGR approximation since it has a pre-exponential factor $\left(A \equiv|a|^{2} \gtrsim 1\right)$ and an exact rate of decay $\Gamma_{0}$, i.e., this result is a self-consistent Fermi Golden Rule (SC-FGR). This term is the dominant one for a wide range of times, leading to

$$
P_{00}(t) \approx A \exp \left(-2 \Gamma_{0} t / \hbar\right)
$$

By analogy with the self-diffusion process in a classical Markov chain, the exponential in Eq. (17) is identified with a pure survival amplitude. Within the same analogy, the second term (the integration path contribution) will be called return amplitude. The "quantum diffusion" described by this term dominates for long times and brings out the details of the spectral structure of the environment.

\section{A. Short time regime}

The second term of Eq. (7) is fundamental for the normalization at very short times. Both terms combine to provide the initial quadratic decay required by the perturbation theory

$$
P_{00}(t)=1-\left\langle\left(\varepsilon-\varepsilon_{r}\right)^{2}\right\rangle_{N_{0}} t^{2} / \hbar^{2}+\cdots,
$$

where $\left\langle\left(\varepsilon-\varepsilon_{r}\right)^{2}\right\rangle_{N_{0}}$ is the second moment of the LDoS $N_{0}(\varepsilon)$. This expansion holds up to the characteristic time $t_{S}$. Let us consider a single state of energy $\varepsilon_{0}$ coupled by $V_{0, j}$ to an environment defined by $\mathrm{N}$ states of energy $\varepsilon_{j}$ spread over a bandwidth $B$, as shows Fig.(2 - $a)$.

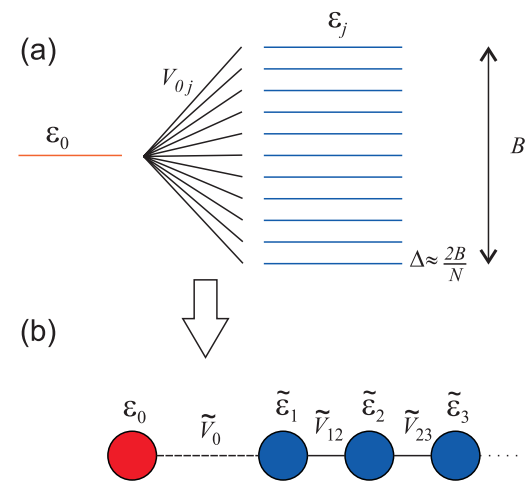

FIG. 2: (color online) (a) Single state of energy $\varepsilon_{0}$ coupled by $V_{0 j}$ to $\mathrm{N}$ states of energy $\varepsilon_{j}$, spread over a bandwidth $B$ with mean-level space of $\Delta \approx 2 B / N$, that defines the environment. (b) Equivalent semi-infinite chain with $\varepsilon_{0}, \widetilde{V_{0}}, \widetilde{\varepsilon_{1}}$, defined in text.

A semi-infinite chain can be obtained from this system by using the recursion method [12], a variant of Lanczos tridiagonalization scheme, as is shown in Fig.(2-b). The first two states are:

$$
\begin{aligned}
& \widetilde{\varepsilon_{0}}=\varepsilon_{0} ; \quad \widetilde{|0\rangle}=|0\rangle \\
& \widetilde{\varepsilon_{1}}=\frac{\sum_{j=1}^{N} V_{j, 0} \varepsilon_{j}}{{\widetilde{V_{0}}}^{2}} ; \widetilde{1\rangle}=\frac{\sum_{j=1}^{N} V_{0, j}|j\rangle}{\widetilde{V_{0}}} \\
& \widetilde{V_{0}}=\sqrt{\sum_{j}^{N}\left|V_{0, j}\right|^{2}}
\end{aligned}
$$

Here, the local second moment of the Hamiltonian is ${\widetilde{V_{0}}}^{2}$, leading to

$$
P_{00}(t)=1-{\widetilde{V_{0}}}^{2} t^{2} / \hbar^{2}+\cdots
$$

There is a simple expression extrapolating Eqs. (13) and (8), [13]:

$$
P_{00}(t) \approx \exp \left[\left(1-\sqrt{1+\left(t / t_{S}\right)^{2}}\right) 2 \Gamma_{0} t_{S} / \hbar\right]
$$

with,

$$
t_{S}=\hbar \frac{\Gamma_{0}}{{\widetilde{V_{0}}}^{2}}
$$


This yields Eq. (13) for $t \ll t_{S}$ at the lowest order. In contrast, for $t \gg t_{S}$ it yields the SC-FGR of Eq. (8), with $A \approx \exp \left(2 \Gamma_{0}^{2} / \widetilde{V}_{0}^{2}\right)$ valid for $\left|\widetilde{V}_{0}\right| \ll B$. Therefore, as was remarked by Pascazio et al. [10], the upper limit for the quadratic behavior is not $\hbar / \widetilde{V}_{0}$, as one might expect, but rather the much shorter time $t_{S}$. A useful interpretation of $t_{S}$ can be drawn from the Green's function [14]:

$$
G_{00}^{R}(\varepsilon)=\frac{1}{\varepsilon-\varepsilon_{0}-\widetilde{V}_{0}^{2} G_{\widetilde{11}}^{R(0)}(\varepsilon)},
$$

where $G_{\widetilde{1} 1}^{R(0)}(\varepsilon)$ corresponds to a semi-infinite chain in absence of 0th-site. Taking $G_{\widetilde{11}}^{R(0)}\left(\varepsilon=\varepsilon_{0}\right)$ Eq.(16) gives the FGR: $\Gamma_{F G R} \approx \pi \widetilde{V}_{0}^{2} N_{\widetilde{1}}^{(0)}\left(\varepsilon_{0}\right)$. Replacing it in Eq. (15), we get

$$
t_{S} \approx \hbar \pi N_{\widetilde{1}}^{(0)}\left(\varepsilon_{0}\right)
$$

only determined by $N_{\widetilde{1}}^{(0)}\left(\varepsilon_{0}\right)$, the LDoS at the 1 st-site of the unperturbed environment, evaluated at $\varepsilon_{0}$. In turns, $\hbar N_{\widetilde{1}}^{(0)}\left(\varepsilon_{0}\right)$ represents the time scale [14] at which an excitation built from the decay, decays into the rest of the environment. Therefore, the return to the 0th-site, required to build up the quadratic decay, becomes less appreciable than the escape towards the chain, leading to the fast exponential decay of the survival probability.

\section{B. Long time regime: Survival collapse}

For long times, only small values of $\varepsilon^{\prime}$ contribute to the integral of the second term in Eq.(77). This restricts the integration to a range near the band-edges. Then, taking into account Eq.(4) and performing the Fourier transform retaining only the Van Hove singularities at these edges, we get the power law decay at long times. This second term dominates $P_{00}(t)$ because its decay is slower than the exponential one. The relative participation on the LDoS at each edge is $\beta=\left[\left(\varepsilon_{r}-\varepsilon_{L}\right)^{2}+\Gamma_{0}^{2}\right] /\left[\left(\varepsilon_{U}-\varepsilon_{r}\right)^{2}+\Gamma_{0}^{2}\right]$. Collecting both edge contributions gives

$$
\begin{aligned}
P_{00}(t) & \approx\left[1+\beta^{2}-2 \beta \cos (B t / \hbar)\right] \\
& \times\left|\int \mathrm{d} \varepsilon^{\prime} \mathrm{e}^{-\varepsilon^{\prime} t / \hbar} N_{0}\left(\varepsilon_{L}-\mathrm{i} \varepsilon^{\prime}\right)\right|^{2} .
\end{aligned}
$$

This means that the long time behavior is just the power law multiplied by a factor containing a modulation with frequency $B / \hbar$. Eq. (77) shows that the survival amplitude of the local excitation recognizes two alternative pathways: the pure survival (pole contribution), and the returning pathways where the excitation has decayed and explored the environment. Then, there is an interference term that becomes important when both amplitudes are of the same order. It is precisely at this cross-over time $t_{R}$ between the exponential regime and the power law regime when the interference term can produce a survival collapse, i.e., $P_{00}(t)$ nearly cancels out. This effect is seen as a pronounced dip in Fig. 3.

We also note that if the unperturbed energy state $\varepsilon_{0}$ is exactly at the center of the band, $\beta=1$, the pure return probability presents periodicals zeros barely compensated by the small pure survival probability. This should not be confused with the survival collapse discussed above, which may yield an exact zero in $P_{00}(t)$.

\section{SEMI-INFINITE CHAIN: EXACT SOLUTION}

Let us focus on a tight-binding Hamiltonian shown in Fig. (2-b) with hoppings $\widetilde{V}_{0}=V_{0}, \widetilde{V}_{j, j+1}=V$ and site energies $\varepsilon_{0}$ and $\varepsilon_{j}=2 V$ for $j>0$ :

$$
\begin{aligned}
\mathcal{H} & =|0\rangle \varepsilon_{0}\langle 0|-\left(|0\rangle V_{0}\langle 1|+\text { c.c. }\right) \\
& +\sum_{n}(|n\rangle 2 V\langle n|-| n\rangle V\langle n+1|+\text { c.c. }) .
\end{aligned}
$$

This defines a continuous spectrum $\left[\varepsilon_{L}=0, \varepsilon_{U}=4 V=\right.$ $B]$ and a well defined resonance for $V_{0} \ll V$. We first summarize the results in Ref. [4]. The LDoS factorizes as a pure Lorentzian around $\varepsilon_{r} \pm \mathrm{i} \Gamma_{0}$, and $N_{1}^{(0)}(\varepsilon)$ :

$$
N_{0}(\varepsilon)=\frac{V^{2}}{\Gamma_{c}} \frac{\Gamma_{0}}{\left(\varepsilon_{r}-\varepsilon\right)^{2}+\Gamma_{0}^{2}} N_{1}^{(0)}(\varepsilon),
$$

with

$$
\begin{aligned}
N_{1}^{(0)}(\varepsilon) & =\frac{16 \Gamma(\varepsilon)}{\pi B^{2}} \theta(|\varepsilon-2 V|) ; \Gamma(\varepsilon)=\frac{\sqrt{\varepsilon} \sqrt{B-\varepsilon}}{2} \\
\varepsilon_{r} & =\varepsilon_{0}+\Delta_{0} ; \Delta_{0}=\frac{V_{0}^{2}}{V^{2}-V_{0}^{2}} \frac{\varepsilon_{0}-2 V}{2}, \\
\Gamma_{0} & =\frac{V_{0}^{2}}{V^{2}-V_{0}^{2}} \Gamma_{c} ; \Gamma_{c}=\sqrt{V^{2}-V_{0}^{2}-\left(\frac{\varepsilon_{0}-2 V}{2}\right)^{2}} .
\end{aligned}
$$

The solution of Eq.(4) results in:

$$
P_{00}(t) \approx\left\{\begin{array}{c}
1-\left(V_{0} t / \hbar\right)^{2}, t<t_{S} \\
A \exp \left(-2 \Gamma_{0} t / \hbar\right), t_{S}<t<t_{R} \\
C\left[1-\frac{2 \beta}{1+\beta^{2}} \sin (B t / \hbar)\right]\left[\hbar /\left(\Gamma\left(\varepsilon_{r}\right) t\right)\right]^{3}, t_{R}<t
\end{array}\right.
$$

with $\beta$ as defined in section 2 and

$$
\begin{aligned}
& A=\frac{\sqrt{\varepsilon_{r}^{2}+\Gamma_{0}^{2}} \sqrt{\left(B-\varepsilon_{r}\right)^{2}+\Gamma_{0}^{2}}}{4 \Gamma_{c}^{2}}, \\
& C=\frac{V_{0}^{4} V \Gamma\left(\varepsilon_{r}\right)^{3}\left(1+\beta^{2}\right)}{4 \pi\left(V^{2}-V_{0}^{2}\right)^{2}\left(\Gamma_{0}^{2}+\varepsilon_{r}^{2}\right)^{2}} .
\end{aligned}
$$

Notice that the cubic power law decay at long times follows from the $\sqrt{\varepsilon}$ dependence of the LDoS near the band 
edge (see inset of Fig. (10)). Fig. (3) shows Eq. (24) for $V_{0} / V=0.4$ and $\varepsilon_{0} / V=1$. One obtain an alternative representation of Eq. (77) by introducing an effective decay rate $\Gamma_{\text {eff }}(t)=-\hbar /(2 t) \ln P_{00}(t)$ [10] whose deviation from $\Gamma_{0}$ is a signature of non-exponential decay. This is shown in the inset of Fig. (3). There, the survival collapse is a pronounced peak in $\Gamma_{\text {eff }}(t)$.

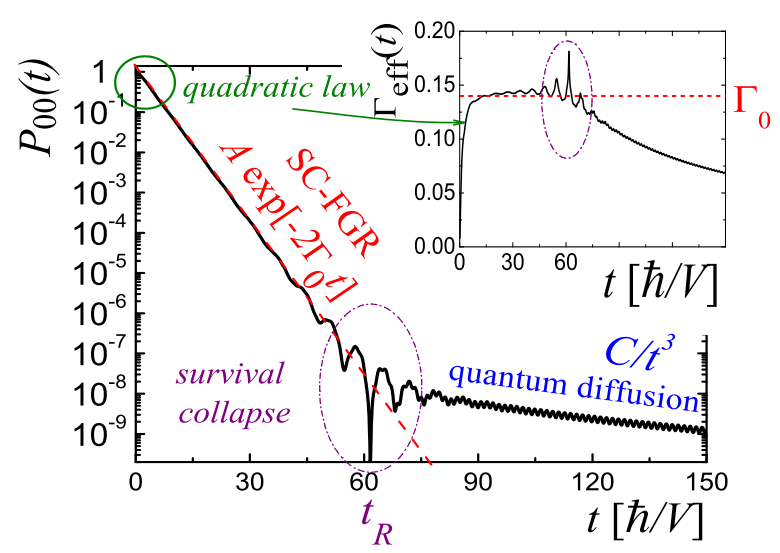

FIG. 3: (color online) Survival probability for a semi-infinite chain with $\varepsilon_{0} / V=1, V_{0} / V=0.4$, that leads to a resonance in $\varepsilon_{r} / V=0.9, \Gamma_{0} / V=0.14$. The inset shows $\Gamma_{\text {eff }}(t)$ (solid line) and $\Gamma_{0}$ (dashed line).

In order to obtain the characteristic time $t_{R}$ in the weak coupling limit we solve iteratively the equality between the exponential and the power law decay (averaged in a period), starting with $\hbar / 2 \Gamma_{0}$. Since, for $\varepsilon_{0}$ close to the center of the band $\sqrt{A / C} \approx \sqrt{32 \pi} V / \Gamma_{0}$ and $\Gamma\left(\varepsilon_{r}\right) \approx V$, we obtain

$$
t_{R}^{(0)}=a_{1} \frac{\hbar}{\Gamma_{0}} \ln \left(a_{2} \frac{B}{4 \Gamma_{0}}\right),
$$

where $a_{1}, a_{2} \gtrsim 1$ are constants that depend on the Van Hove singularity $N_{0}(\varepsilon) \sim\left(\varepsilon-\varepsilon_{L}\right)^{\nu}$ and other details of the model. For a semi-infinite chain $a_{1}=\nu+2=5 / 2$ and $a_{2}=\sqrt[5]{4 \pi} \sim 1.6$. By choosing the parameters $V_{0}, \varepsilon_{0}$ as above, this characteristic time results in $t_{R}^{(0)} \approx 41$ $[\hbar / V]$, which is somewhat smaller than the exact time $t_{R} \approx 62[\hbar / V]$. Just the next order of iteration gives a much better approximation $t_{R}^{(1)} \approx 67[\hbar / V]$. Also, by using these parameters, Eq. (17) results in $t_{S} \approx 0.8[\hbar / V]$ which is a good bound for the short time scale.

In the range of quadratic decay, recursive projective measurement of state $|0\rangle$ at a time interval $\tau_{\phi}$ would produce a deceleration of the decay, i.e., a Quantum Zeno effect (see, for example, Fig. 1 in 16$]$ ). Our results above provides a convenient upper bound, $\tau_{\phi}<t_{S}$, for this time scale.

The survival collapse can also occur at the strong coupling limit at the cross-over between the short time regime and the power law decay. Fig. (44) shows $P_{00}(t)$ and $\Gamma_{\text {eff }}(t)$ for $\varepsilon_{0} / V=1.8$ and $V_{0} / V=0.77$, which yields to $t_{R} \approx 6.8[\hbar / V]$. In this case, recursive projective measurement at a time interval $\tau_{\phi} \approx t_{R}$ can make the survival probability much smaller. Then the survival collapse enables an acceleration of the decay induced by repetitive observations, i.e., an anti-Zeno effect [10].

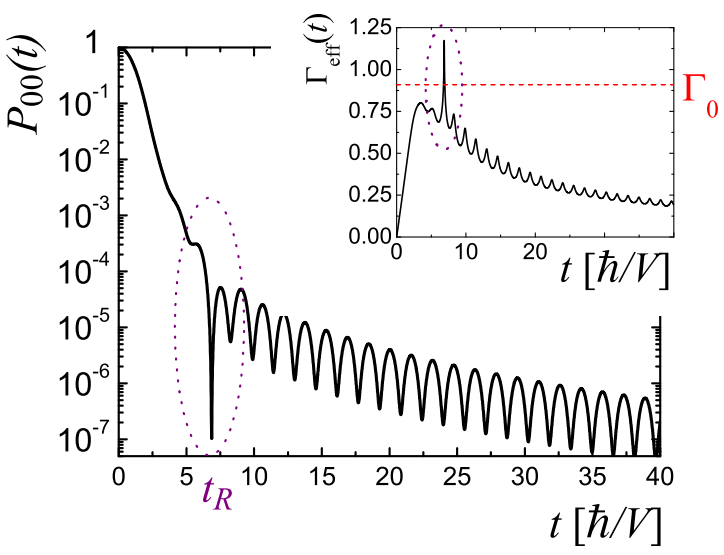

FIG. 4: (color online) Survival probability for a semi-infinite chain with $\varepsilon_{0} / V=1.8, V_{0} / V=0.77$. The inset shows $\Gamma_{e f f}(t)$ (solid line) and $\Gamma_{0}$ (dashed line).

\section{CONCLUSIONS}

We studied the dynamics of a local excitation in a system in which full memory effects at the environment are included. We obtain the time limits where the nonexponential behavior of the survival probability shows up. The evolution starts with the expected quadratic decay, which holds up to a time $t_{S}$ (Eq. (17)) determined by the density of the first state of the environment in absence of the initial state. This time gives an upper bound to the interval at which repetitive projection measurements leads to a Quantum Zeno effect. In the weak coupling limit the decay follows the usual FGR exponential, but with a corrected rate and a pre-exponential factor, i.e., the SC-FGR. At long times we get a power law decay controlled by non-Markovian return processes. We also visualized a survival collapse at time $t_{R}$ (Eq. (27)) as a destructive interference between the pure survival amplitude and the return amplitude. This last arises from pathways that explore the environment before returning. Given that a survival collapse occurs, one can use repetitive projective measurements with a period $\tau_{\phi} \approx t_{R}$ to achieve an anti-Zeno effect.

The authors acknowledge financial support from CONICET, SeCyT-UNC and ANPCyT as well as hospitality of Abdus-Salam ICTP. 
[1] S. R. Wilkinson, C. F. Bharucha, M. C. Fischer, K. W. Madison, P. R. Morrow, Q. Niu, B. Sundaram, M. G. Raizen, Nature 387 (1997) 575.

[2] L. Khalfin, Sov. Phys. JETP 6 (1958) 1053; G. GarcíaCalderón, J. L. Mateos and M. Moshinsky, Phys. Rev. Lett. 74 (1995) 337.

[3] A. G. Kofman, G. Kurizki, B. Sherman, J. Mod. Opt. 41(1994) 353; P. Facchi, S. Pascazio, Phys. A 271 (1999) 133.

[4] E. Rufeil-Fiori, H. M. Pastawski, Chem. Phys. Lett. 420 (2006) 35.

[5] G. García-Calderón, R. Romo, J. Villavicencio, Phys. Rev. 76 (2007) 035340.

[6] S. Longhi, Phys. Rev. Lett. 97 (2006) 110402.

[7] Z. L. Mádi, B. Brutsher, T. Schulte-Herbrüggen, R. Brüschweiler, R. R. Ernst, Chem. Phys. Lett. 268 (1997) 300.

[8] S. I. Doronin, I. I. Maksimov, E. B. Fel'dman, J. of Exp. and Theor. Phys. 91 (2000) 597; P. Capellaro, C. Ramanathan, D. G. Cory, Phys. Rev. Lett. 99 (2007)
250506; E. Rufeil-Fiori, C. M. Sanchez, F. Y. Oliva, H. M.Pastawski, P. R. Levstein, Phys. Rev. A 79 (2009) 032324.

[9] B. Misra, E. C. G. Sudarshan, J. Math. Phys. 18 (1977) 756.

[10] P. Facchi, H. Nakazato, S. Pascazio, Phys. Rev. Lett. 87 (2001) 2699.

[11] M. C. Fischer, B. Gutierrez-Medina, M. G. Raizen, Phys. Rev. Lett. 87 (2001) 040402.

[12] R. Haydock, V. Heine, M. J. Kelly, J. Phys. C 5 (1972) 2845.

[13] V. V. Flambaum, F. M. Izrailev, Phys. Rev. E 64 (2001) 026124.

[14] H. M. Pastawski, E. Medina, Rev. Mex. de Fís. 47 (2001) 1 .

[15] A. D. Dente, R. Bustos-Marun, H. M. Pastawski, Phys. Rev. A 78 (2008) 062116.

[16] H. M. Pastawski, G. Usaj, Phys. Rev. B 57 (1998) 5017. 